\title{
ENDOSCOPIC TREATMENT OF POST-LIVER TRANSPLANTATION ANASTOMOTIC BILIARY STRICTURE: systematic review and meta-analysis
}

\author{
Lucas Souto NACIF', Wanderley Marques BERNARDO², Luca BERNARDO², \\ Wellington ANDRAUS ${ }^{1}$, Lucas TORRES ${ }^{1}$, Eleazar CHAIB ${ }^{1}$, \\ Luiz Carneiro D'ALBUQUERQUE' ${ }^{1}$ and Fauze MALUF-FILHO'
}

\begin{abstract}
Context - Biliary strictures after liver transplantation are recognized as its Achilles' heel. The strictures are classified in anastomotic and ischemic or non-anastomotic biliary strictures, and they figure among the most common complications after liver transplantation. There are some treatment options including balloon dilation, the placement of multiple plastic stents and the placement of self-expandable metal stents and all of them seem to have good results. Objective - The aim of this study was to systematically review the literature concerning the results of the endoscopic treatment of anastomotic biliary strictures after liver transplantation. Method - A systematic review of the literature was performed on the management of anastomotic biliary strictures post- orthotopic liver transplantation. The Medline-PubMed, EMBASE, Scielo-LILACS, and Cochrane Databases were electronically searched from January 1966 to April 2013. Results - No well-designed randomized controlled trial was found. Most studies were retrospective or prospective comparisons in design. One study (86 patients) compared the endoscopic and the percutaneous accesses. The sustained clinical success rates were similar but the treatment duration was longer in the percutaneous group access. Two studies (56 patients) compared balloon dilation with balloon dilation and multiple plastic stents. There were no differences concerning sustained clinical success and complication rates. Conclusion - Balloon dilation is as effective as balloon dilation plus multiple plastic stenting for the resolution of the anastomotic biliary strictures. Well-designed randomized trials are still needed to compare balloon dilation versus multiple plastic stenting versus metallic stenting.
\end{abstract}

HEADINGS - Liver transplantation. Constriction. Review. meta-analysis. Gastrointestinal endoscopy.

\section{INTRODUCTION}

Orthotopic liver transplantation (OLT) is a wellaccepted treatment of end-stage liver disease. Biliary fistula, anastomotic biliary strictures $(\mathrm{ABS})^{(5,17)}$ and ischemic biliary lesions ${ }^{(18)}$ figure among the most common complications after OLT.

Considering specifically ABS there are some treatment options including balloon dilation, the placement of multiple plastic stents (MPS) and the placement of self-expandable metal stents ${ }^{(6,9,10,15,18)}$. These modalities can be employed individually or in association. Furthermore the biliary access can be achieved by the percutaneous route (PTC) or retrogradely by the endoscopic route (ERC). It is recognized that ERC is the approach of choice when the complication involves an end-to-end biliary anastomosis. However it is not clear which treatment modality presents the best result. On the other hand when less invasive treatment fails surgical hepaticojejunal anastomosis is usually indicated.

Despite the rather high incidence of ABS after OLT and the lack of a well-established treatment algorithm there is a paucity of randomized controlled trials and systematic reviews on the subject. Thus, the aims of this study were to systematically review the literature concerning ABS after OLT and to organize a meta-analysis with the results.

Declared conflict of interest of all authors: none

Authors certificate that there are no financial competing interests

Research performed at: Departamento de Gastroenterologia, Divisão de Transplante Hepático e Gastrointestinal, Laboratório de Investigação Médica, Faculdade de Medicina, Universidade de São Paulo - FMUSP.

'Departamento de Gastroenterologia, Divisão de Transplante Hepático e Gastrointestinal, Laboratório de Investigação Médica, LIM-37 FMUSP; ${ }^{2}$ Projeto Diretrizes Associação Médica Brasileira. São Paulo, SP, Brasil.

Correspondence: Fauze Maluf Filho Divisão de Transplante Hepático e Gastrointestinal. Departamento de Gastroenterologia. Faculdade de Medicina, Universidade de São Paulo. CEP 05403-900, São Paulo, SP, Brasil.E-mail: fauze.maluf@terra.com.br 


\section{METHODS}

\section{Study identification and selection}

A systematic review of the literature was performed on the management of ABS post-OLT. The Medline-PubMed, EMBASE, Scielo-LILACS, and Cochrane Databases were electronically searched from January 1966 to April 2013.

The terms for each group were sought in combination using the "OR" boolean operator. The results for the search terms which formed the "P" (Patients) group were combined with the result for the search which formed the "I" (Intervention) group, using the "AND" boolean operator, and subsequently with the "exclusion key words" using the "NOT" boolean operator (Figure 1).

\begin{tabular}{|ll|}
\hline & Terms search PubMed database \\
& (Post-liver transplantation OR Liver \\
transplantation OR Liver transplant & OR Hepatic Transplantation OR \\
Patients OR /AND & Liver Grafting AND Constrictions, \\
& Pathologic OR Stricture OR Strictures \\
& OR Stenose OR Stenoses OR Stenosis) \\
\hline & AND (percutaneous transhepatic \\
biliary drainage OR percutaneous & interventions OR percutaneous \\
treatment OR drainage OR \\
percutaneous OR Stents OR \\
Cholangiopancreatography, Endoscopic \\
Retrograde OR ERCP OR \\
Cholangiography) \\
Intervention OR / AND \\
NOT (artery OR hepatic artery) \\
\hline Exclusion NOT
\end{tabular}

FIGURE 1. The terms used on PubMed database search were developed using patient, intervention, comparison or control, outcome (PICO) structure

The MEDLINE search was performed through PubMed (www.ncbi.nlm.nih.gov/pubmed) and was adapted by using the terms and Mesh-terms (Post-liver transplantation or Liver transplantation or Liver transplant or Hepatic Transplantation or Liver Grafting) and (Constrictions, Pathologic or Stricture* or Stenose* or Stenosis*) and (Liver Transplantation or Hepatic Transplantation or Liver Grafting) and (Stents or Cholangiopancreatography, Endoscopic Retrograde or ERCP or Cholangiography) or (Post-liver transplantation or Liver transplantation or Liver transplant or Hepatic Transplantation or Liver Grafting) and (Constrictions, Pathologic or Stricture* or Stenose* or Stenosis*) or ((Post-liver transplantation or Liver transplantation or Liver transplant or Hepatic Transplantation or Liver Grafting) and (percutaneous transhepatic biliary drainage or percutaneous interventions or percutaneous treatment or drainage or percutaneous). This initial search was followed by a more specific and detailed one in accordance with our patient, intervention, comparison or control, outcome (PICO) structure using these terms and Mesh-terms observed in Figure 1. The same strategy was used in the Embase (www.embase. com) and LILACS (http://lilacs.bvsalud.org) databases. The Cochrane Library Database was searched for both registered and already published systematic reviews on the treatment of ABS after OLT.

We included clinical studies involving adult and pediatric patients who were treated for ABS after OLT (either cadaveric or living donor organs). The quality of the studies was evaluated by two independent researchers (LSN and WMB). In case of disagreement the researchers held a consensus meeting to reach a final decision.

This study was approved by the Institutional Review Board.

\section{Inclusion and exclusion criteria}

The research questions and the inclusion and exclusion criteria were developed using a PICO structure. Only comparative clinical studies within the limits of the PICO structure were included. We excluded studies with ischemic biliary lesions, artery constriction and surgical treatment.

\section{Critical evaluation and quality of studies}

A critical evaluation of the quality of the studies was conducted, aiming to evaluate the strength of evidence and the validity of their inclusion in this review. The level of evidence and the New Castle score (Ottawa Quality Assessment Cohort Studies) ${ }^{(16)}$ was used, and only studies with a score equal to or greater than six were included in this review.

\section{Statistical analysis}

The measures of effectiveness of each treatment were expressed in absolute numbers and respective frequencies, i.e., the absolute risk. For the meta-analysis the results obtained from the included papers were compared by using the difference in absolute risks, adopting a confidence interval of $95 \%$ and a statistical significant level of $P<0.05$.

\section{Heterogeneity and sensitivity analysis}

Inconsistencies between studies were estimated using the chi-squared test for heterogeneity, and quantified using the I 2 test. A value above $50 \%$ was considered significant. A sensitivity analysis was performed, including only studies that obtained results with power $>80 \%$.

\section{RESULTS}

\section{Study selection}

By using the search strategy, 3655 articles were initially retrieved. Their abstracts were reviewed and 13 studies were selected according to the inclusion criteria (Figure 2). Finally we extracted the data from 5 comparative studies to calculate 
the meta-analysis (Figure 3). No systematic reviews or metaanalysis on the treatment of ABS after OLT were found in the Cochrane Library.

\section{Quality assessment of the studies}

The data of the selected primary studies selection are described in Figure 3.

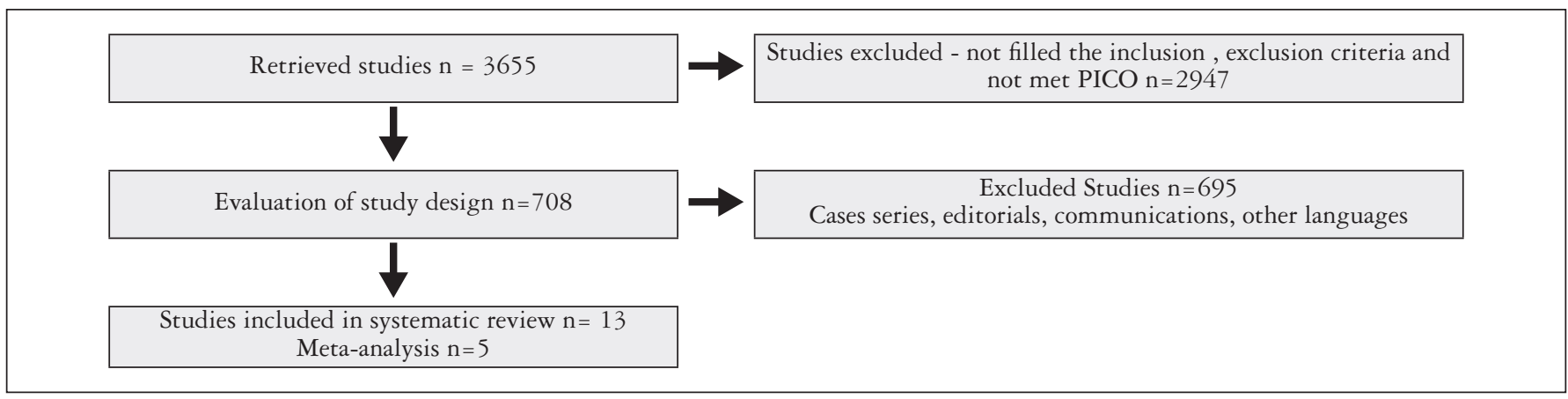

FIGURE 2. Diagram of this systematic review showing the initial steps to select the articles and the included and excluded citations.

\begin{tabular}{|c|c|c|c|c|}
\hline Study & Population & Intervention & Comparison & Outcome \\
\hline $\begin{array}{l}\text { Chang JH et al. }{ }^{(1)} \\
2012\end{array}$ & $\begin{array}{l}\text { Patients with a biliary } \\
\text { stricture after living } \\
\text { donor LT }\end{array}$ & $\begin{array}{l}\text { Rendezvous technique - } \\
\text { percutaneous transhepatic } \\
\text { biliary drainage catheters for } \\
\text { inside stents }\end{array}$ & Guidewire group vs KMP catheter & $\begin{array}{l}\text { Rendezvous technique use KMP } \\
\text { catheter was a fast and safe }\end{array}$ \\
\hline $\begin{array}{l}\text { Zoepf T et al. }{ }^{(18)} \\
2012\end{array}$ & $\begin{array}{l}\text { Adult patients underwent } \\
\text { LT (ischemic-type biliary } \\
\text { lesions) }\end{array}$ & Endoscopic intervention & $\begin{array}{l}\text { BD vs combination implantation } \\
\text { of a } \mathrm{BD}+\mathrm{EP}\end{array}$ & $\begin{array}{l}\mathrm{BD}+\mathrm{EP} \text { was successful in } 5 \text { of } 16 \\
(31 \%) \text { and } \mathrm{BD} \text { alone in } 30 \text { of } 32 \\
\text { patients }(91 \% ; P=0.0027)\end{array}$ \\
\hline \begin{tabular}{|l|} 
Tarantino I et \\
al. ${ }^{(15)} 2012$ \\
\end{tabular} & Benign biliary stricture & $\begin{array}{l}\text { Fully covered self-expandable } \\
\text { metal stents }\end{array}$ & $\begin{array}{l}\text { After endoscopic therapy vs no } \\
\text { previous endoscopic treatment }\end{array}$ & $\begin{array}{l}\text { Effective for refractory benign biliary } \\
\text { strictures }\end{array}$ \\
\hline $\begin{array}{l}\text { Giampalma E et } \\
\text { al. }{ }^{(3)} 2012\end{array}$ & $\begin{array}{l}\text { Post-LT biliary stricture } \\
\text { (BSs) }\end{array}$ & \begin{tabular}{|l|} 
Percutaneous treatment \\
Bilioplasty and were refractory \\
to bilioplasty, metallic stents \\
\end{tabular} & $\begin{array}{l}\text { Ischemic and nonischemic biliary } \\
\text { stenoses }\end{array}$ & $\begin{array}{l}\text { Ischemic stricture needs closer follow } \\
\text { up }\end{array}$ \\
\hline $\begin{array}{l}\text { Li QY et al. }{ }^{(11)} \\
2011\end{array}$ & AS after LT & ERCP & $\begin{array}{l}\text { Intrahepatic biliary dilation vs } \\
\text { AS without intrahepatic biliary } \\
\text { dilation }\end{array}$ & $\begin{array}{l}\text { ERCP is not effective in AS without } \\
\text { intrahepatic biliary dilation after LT }\end{array}$ \\
\hline $\begin{array}{l}\text { Kim J et al. } \\
2010\end{array}$ & $\begin{array}{l}\text { Biliary anastomotic } \\
\text { strictures following LDLT }\end{array}$ & Percutaneous treatment & $\begin{array}{l}\text { Covered retrievable stent placement } \\
\text { vs drainage catheter placement } \\
\text { for treating biliary anastomotic } \\
\text { strictures }\end{array}$ & $\begin{array}{l}\text { Covered retrievable stents has an } \\
\text { acceptable clinical success rate with } \\
\text { shorter treatment duration }\end{array}$ \\
\hline $\begin{array}{l}\text { Tabibian JH et } \\
\text { al. }^{(14)} 2010\end{array}$ & $\begin{array}{l}\text { Post LT anastomotic } \\
\text { biliary strictures }\end{array}$ & ERCP & $\begin{array}{l}\text { ERCP with balloon dilation and } \\
\text { maximal stenting }\end{array}$ & $\begin{array}{l}\text { Maximal stenting protocol for ABSs } \\
\text { is effective, safe, rarely associated with } \\
\text { ABS recurrence }\end{array}$ \\
\hline $\begin{array}{l}\text { Gomez CM et al. }{ }^{(4)} \\
2009\end{array}$ & $\begin{array}{l}\text { Biliary complications after } \\
\text { DDLT and to adult LDLT }\end{array}$ & ERCP & $\begin{array}{l}30 \text { LDLT vs } 357 \text { DDLT consecutive } \\
\text { adult recipients with duct-to-duct } \\
\text { biliary reconstruction }\end{array}$ & $\begin{array}{l}\text { Biliary complications were more } \\
\text { frequent after LDLT compared with } \\
\text { DDLT }\end{array}$ \\
\hline $\begin{array}{l}\text { Kobayashi T et } \\
\text { al. }{ }^{(7)} 2009\end{array}$ & $\begin{array}{l}\text { Biliary strictures after } \\
\text { adult living donor liver } \\
\text { transplant }\end{array}$ & $\begin{array}{l}\text { Endoscopic retrograde } \\
\text { cholangiography }\end{array}$ & $\begin{array}{l}\text { Endoscopic approach for endoscopic } \\
\text { dilatation and/or stent placement }\end{array}$ & $\begin{array}{l}\text { Endoscopic treatment after OLT was a } \\
\text { significant risk factor for post-ERCP } \\
\text { pancreatitis }\end{array}$ \\
\hline $\begin{array}{l}\text { Kulaksiz H et al. }{ }^{(9)} \\
2008\end{array}$ & Post-LT biliary strictures & $\begin{array}{l}\text { Endoscopic treatment of } \\
\text { strictures }\end{array}$ & $\begin{array}{l}\text { Balloon dilation vs balloon dilation } \\
\text { plus stenting }\end{array}$ & $\begin{array}{l}\text { Endoscopic balloon dilation alone } \\
\text { was as effective as dilation plus stent } \\
\text { placement }\end{array}$ \\
\hline $\begin{array}{l}\text { Lee SH et al. }{ }^{(10)} \\
2008\end{array}$ & Biliary strictures after LT & ECP and PCP & $\begin{array}{l}\text { ECP and PCP for anastomotic } \\
\text { stricture vs non-anastomotic } \\
\text { stricture }\end{array}$ & $\begin{array}{l}\text { Endoscopic access should be the } \\
\text { preferred first approach in patients } \\
\text { with biliary stricture after LT }\end{array}$ \\
\hline $\begin{array}{l}\text { Pasha SF et al. }{ }^{(12)} \\
2007\end{array}$ & $\begin{array}{l}\text { ABS after deceased donor } \\
\text { LT }\end{array}$ & Endoscopic treatment & $\begin{array}{l}\text { Combined technique of BD and } \\
\text { maximal stent placement }\end{array}$ & Combined therapy was successful \\
\hline $\begin{array}{l}\text { Zoepf } T \text { et al. }{ }^{(17)} \\
2006\end{array}$ & ABS after-LT & Endoscopic treatment & $\mathrm{BD}$ or combined $\mathrm{BD}+\mathrm{EP}$ & $\begin{array}{l}\text { Combination of BD followed by an } \\
\text { increasing number and diameter of } \\
\text { endoprostheses }\end{array}$ \\
\hline
\end{tabular}

FIGURE 3. The PICO structure developed using a population, intervention, comparison and outcome.

The studies selected for meta-analysis are in bold.

LT: liver transplantation; KMP: Kumpe; BD: balloon dilation; EP: plastic endoprosthesis; BS: biliary stricture; AS: anastomotic stricture; ERCP: Endoscopic retrograde cholangiopancreatography; LDLT: living donor liver transplantation; DDLT: deceased-donor liver transplantation; ABS: anastomotic biliary strictures; OLT: orthotopic liver transplantation; ECP: Endoscopic cholangioplasty; PCP: percutaneous cholangioplasty. 
The quality assessment of the studies including study design, level of evidence and New Castle score (Ottawa Quality Assessment Cohort Studies) ${ }^{(16)}$ were demonstrated in Figure 4.

\section{Meta-analysis}

Three meta-analysis calculations involved 5 comparative studies: 1. Endoscopic access (ECP) vs the percutaneous access (PCP) (Table 1); 2. Endoscopic balloon dilation as- sociated with plastic endoprothesis $(\mathrm{BD}+\mathrm{EP})$ vs balloon dilatation alone (BD) (Table 2); 3. Self-expandable metallic stents (SEMS) as salvage therapy vs. SEMS as 1st line treatment deployed by ECP (Table 3 ).

\section{Endoscopic access (ECP) vs Percutaneous access (PCP)}

Only one study compared ECP vs PCP for the treatment of ABS after OLT and it was included in this meta-analysis

\begin{tabular}{|c|c|c|c|}
\hline Study & Design & Level of evidence & New Castle $1 / 2 / 3 / 4 / 5 / 6 / 7 / 8 / 9$ Total: $\geq 6$ \\
\hline Chang JH et al. ${ }^{(1)} 2012$ & Comparative retrospective study & $2 \mathrm{C}$ & $\begin{array}{l}\text { Selection: } * / * / * \\
\text { Comparability:* } \\
\text { Outcome:*/* } \\
\text { Total: } 6\end{array}$ \\
\hline Zoepf T et al. ${ }^{(18)} 2012$ & Comparative retrospective study & $2 \mathrm{C}$ & $\begin{array}{l}\text { Selection: } * / * / * \\
\text { Comparability:*/* } \\
\text { Outcome:*/* } \\
\text { Total: } 7\end{array}$ \\
\hline Tarantino I et al..$^{(15)} 2012$ & Comparative prospective study & 2B & $\begin{array}{l}\text { Selection: } * / * / * \\
\text { Comparability:*/* } \\
\text { Outcome:*/*/* } \\
\text { Total: } 8\end{array}$ \\
\hline Giampalma E et al. ${ }^{(3)} 2012$ & Prospective comparative study & $2 \mathrm{C}$ & $\begin{array}{l}\text { Selection: } * / * / * \\
\text { Comparability:* } \\
\text { Outcome:*/*/* } \\
\text { Total: } 7\end{array}$ \\
\hline Li QY et al. ${ }^{(11)} 2011$ & Prospective comparative study & $2 \mathrm{C}$ & $\begin{array}{l}\text { Selection: } * / * / * \\
\text { Comparability:* } \\
\text { Outcome:*/* } \\
\text { Total: } 6\end{array}$ \\
\hline Kim J et al. ${ }^{(6)} 2010$ & Prospective comparative study & $2 \mathrm{C}$ & $\begin{array}{l}\text { Selection: } * / * / * \\
\text { Comparability:* } \\
\text { Outcome:*/*/* } \\
\text { Total: } 7\end{array}$ \\
\hline Tabibian JH et al. ${ }^{(14)} 2010$ & Comparative retrospective study & $2 \mathrm{C}$ & $\begin{array}{l}\text { Selection: } * / * / * \\
\text { Comparability:* } \\
\text { Outcome:*/*/* } \\
\text { Total: } 7\end{array}$ \\
\hline Gomez CM et al. ${ }^{(4)} 2009$ & Retrospective comparative study & $2 \mathrm{C}$ & $\begin{array}{l}\text { Selection: } * / * / * \\
\text { Comparability:* } \\
\text { Outcome:*/* } \\
\text { Total: } 6\end{array}$ \\
\hline Kobayashi T et al. ${ }^{(7)} 2009$ & Retrospective comparative syudy & $2 \mathrm{C}$ & $\begin{array}{l}\text { Selection: } * / * / * \\
\text { Comparability:* } \\
\text { Outcome:*/*/* } \\
\text { Total: } 7\end{array}$ \\
\hline Kulaksiz H et al. ${ }^{(9)} 2008$ & Prospective comparative study & $2 \mathrm{C}$ & $\begin{array}{l}\text { Selection: } * / * / * \\
\text { Comparability:* } \\
\text { Outcome:*/* } \\
\text { Total: } 6\end{array}$ \\
\hline Lee SH et al. ${ }^{(10)} 2008$ & Comparative study & $2 \mathrm{C}$ & $\begin{array}{l}\text { Selection: } * / * / * \\
\text { Comparability:* } \\
\text { Outcome:*/* } \\
\text { Total: } 6\end{array}$ \\
\hline Pasha SF et al. ${ }^{(12)} 2007$ & Comparative Study & $2 \mathrm{C}$ & $\begin{array}{l}\text { Selection: } * / * / * \\
\text { Comparability:* } \\
\text { Outcome:*/*/* } \\
\text { Total: } 7\end{array}$ \\
\hline Zoepf T et al. ${ }^{(17)} 2006$ & Retrospective Comparative Study & $2 \mathrm{C}$ & $\begin{array}{l}\text { Selection:*/*/* } \\
\text { Comparability:* } \\
\text { Outcome:*/*/* } \\
\text { Total: } 7\end{array}$ \\
\hline
\end{tabular}

FIGURE 4. Quality assessment of the studies - study design, level of evidence and New Castle (Ottawa Quality Assessment Cohort Studies). 
TABLE 1. Endoscopic (ECP) vs Percutaneous (PCP) access

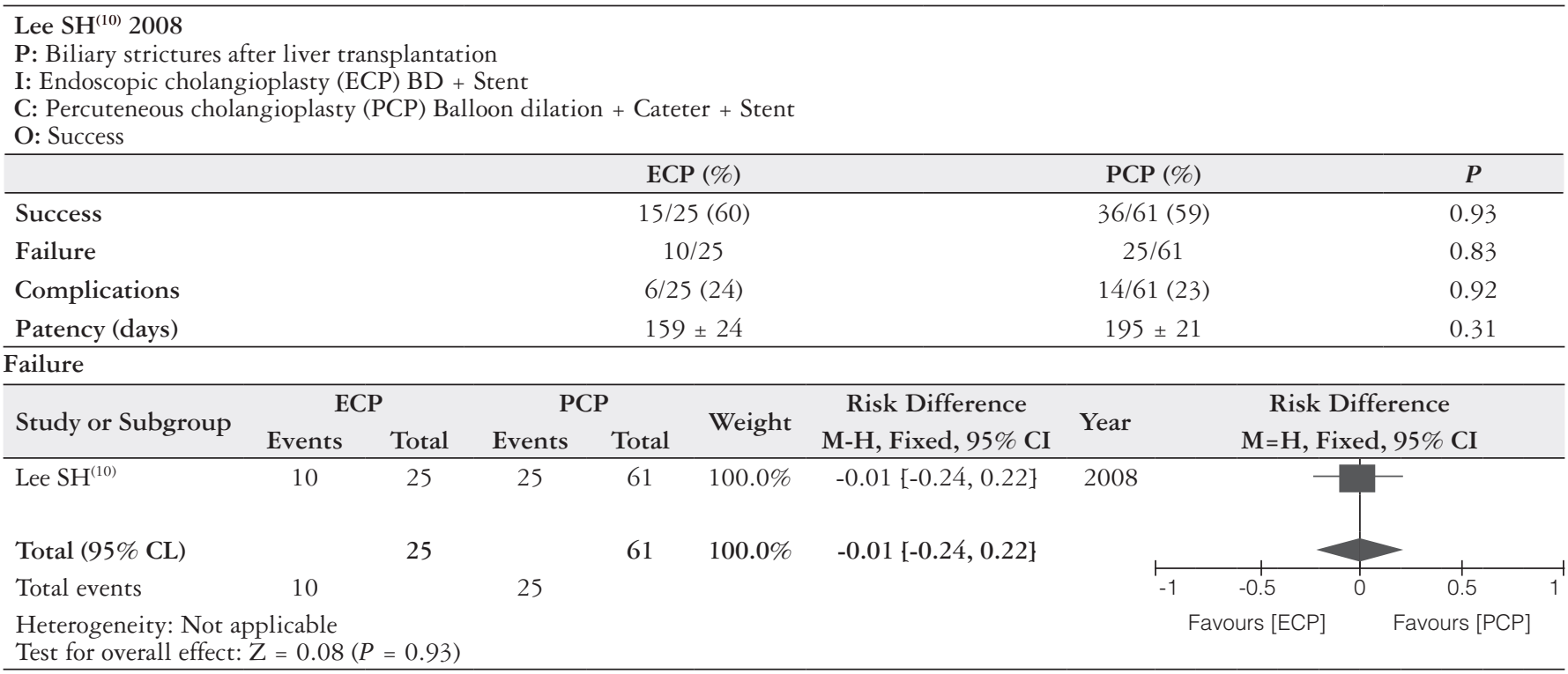

TABLE 2. Balloon dilation and Endoprothesis $(\mathrm{BD}+\mathrm{EP})$ vs. Balloon dilatation (BD) or ERCP.

\begin{tabular}{lccc}
\hline Zoepf $\mathrm{T}^{(18)} 2006$ & & & \\
P: Post Liver Transplant Anastomotic Stricture (PTAS) & & & \\
I: BD & & & \\
C: BD+EP & BD $(\%)$ & BD + EP $(\%)$ & P \\
O: Normalization of Cholestasis & $8 / 9(89)$ & $13 / 15(87)$ & NA \\
\hline & $1 / 9(11)$ & $4 / 13(13)$ & NA \\
\hline Initial success & $5 / 8(62.5)$ & $9 / 60(15)$ & NA \\
Initial failure & $4 / 18(22)$ & $3 / 60$ & NA \\
Recurrence & $3 / 18$ & $5 / 60$ & NA \\
Complications & $0 / 18$ & $120 \mathrm{~d} / 4 \mathrm{~m}$ & NA \\
Cholangitis & $45 \mathrm{~d} / 1.5 \mathrm{~m}$ & &
\end{tabular}

Kulaksiz $\mathrm{H}^{(9)} 2008$ Prospective comparative study

P: Symptomatic biliary strictures after liver transplantation (Anastomotic and Non-anastomotic BS)

I: $\mathrm{BD}$

$\mathrm{C}: \mathrm{BD}+\mathrm{EP}$

O: Dilation success ( 3 months without further endoscopic intervention)

\begin{tabular}{|c|c|c|c|}
\hline & $\mathrm{BD}(\%)$ & $\mathrm{BD}+\mathrm{EP}(\%)$ & $P$ \\
\hline $\begin{array}{l}\text { Initial Success (anastomotic and } \\
\text { non-anastomotic strictures) }\end{array}$ & $12 / 17(70.6)$ & $11 / 15(73.3)$ & n.s. \\
\hline Anastomotic stricture & 10 & 9 & NA \\
\hline Success/Recurrence & $10 / 1$ & $9 / 2$ & NA \\
\hline Non-anastomotic stricture & 4 & 2 & NA \\
\hline Success/Recurrence & $2 / 1$ & - & NA \\
\hline Initial Failure & $5 / 17(29.4)$ & $4 / 15(26.7)$ & NA \\
\hline Recurrence & $2 / 17$ & $2 / 15$ & NA \\
\hline Complications & $4 / 94(4.3)$ & 12/87 (13.6) & $<0.05$ \\
\hline Cholangitis & $2 / 94$ & $4 / 87$ & NA \\
\hline Pancreatitis & $1 / 94$ & $2 / 87$ & NA \\
\hline Mean duration of the treament & $183 \pm 111$ & $153 \pm 57$ & n.s. \\
\hline
\end{tabular}


1.1 Failure - Anastomotic and Non-anastomotic Strictures

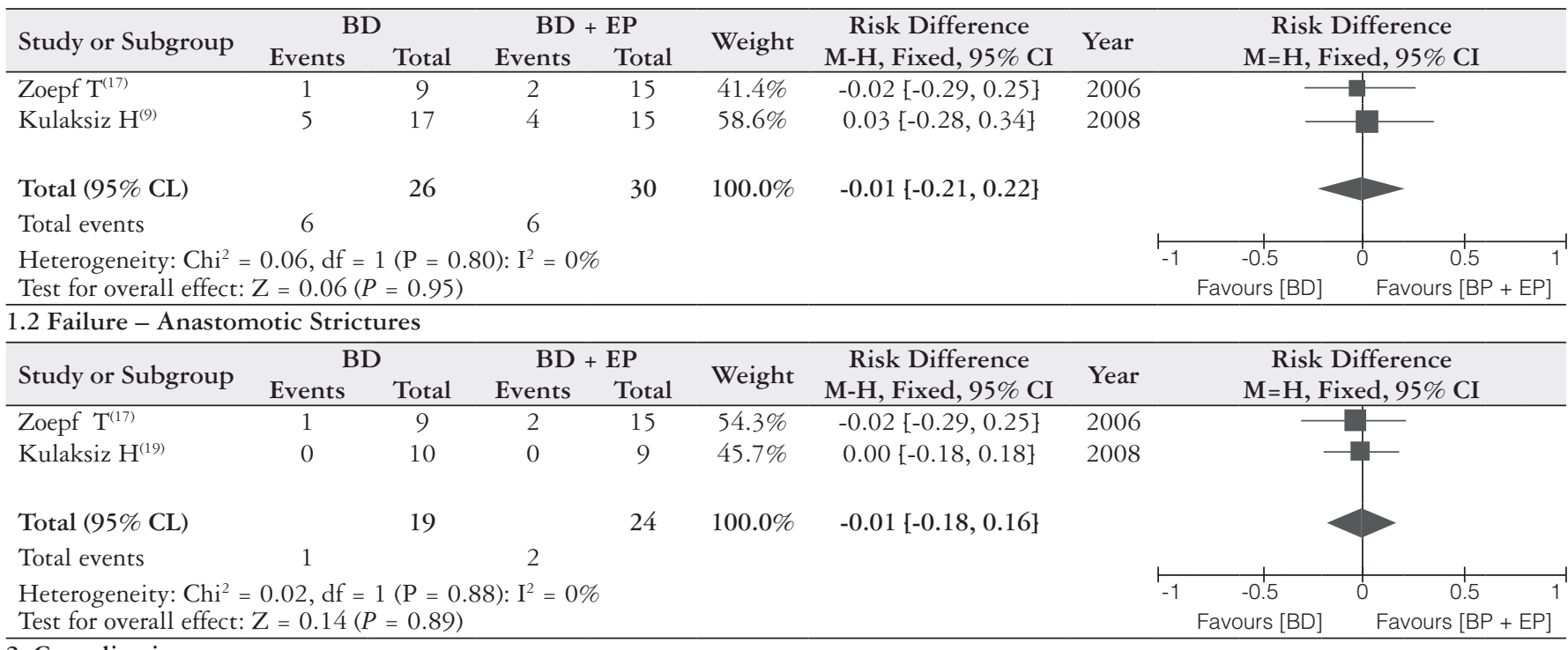

2. Complications

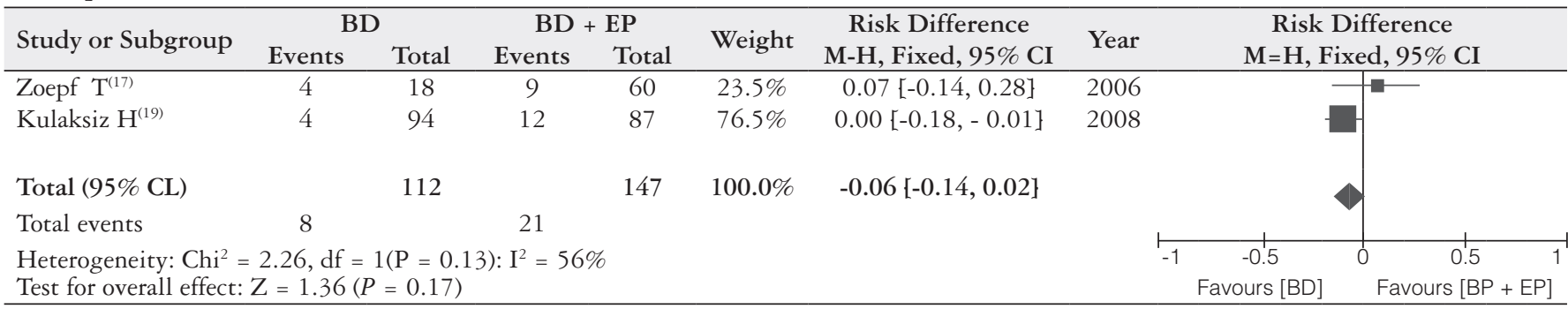

3. Cholangitis

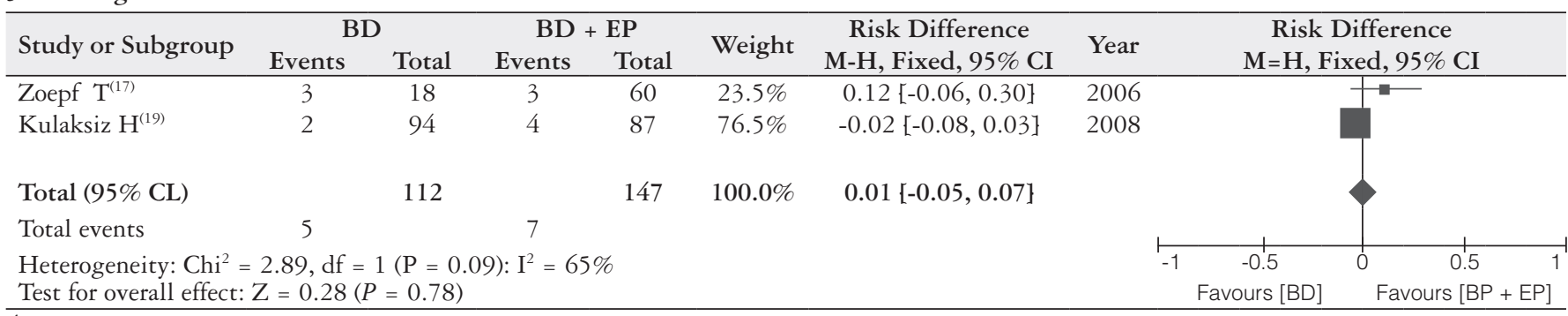

4. Pancreatitis

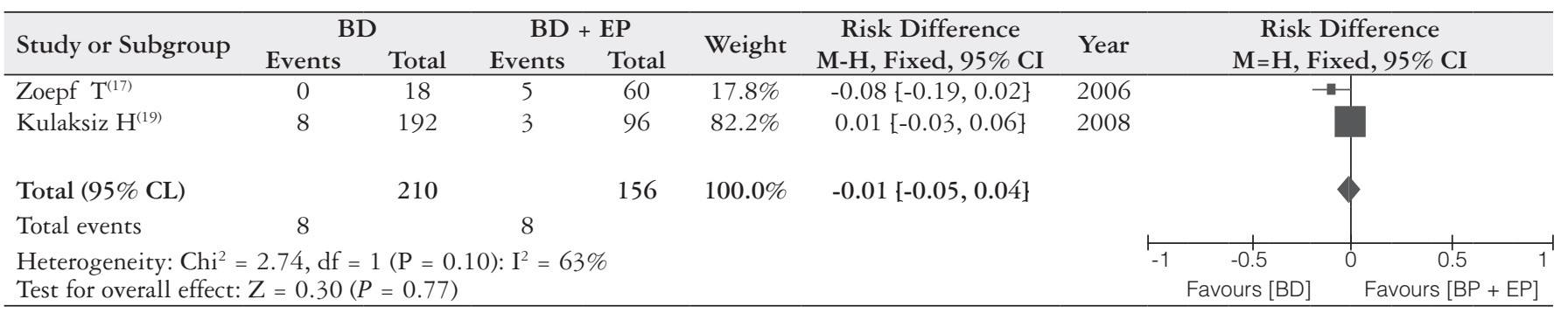

5. Recurrence - Anastomotic Strictures

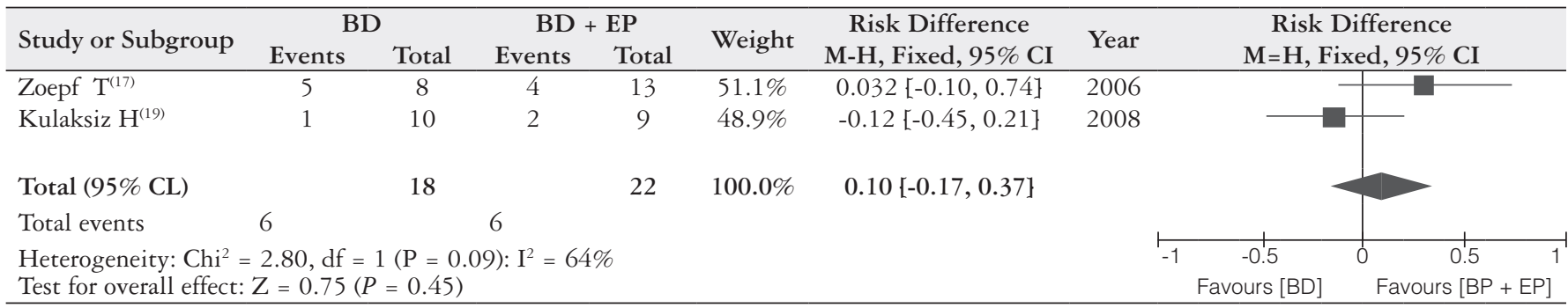


TABLE 3. Self-expandable metallic stents (SEMS) as salvage therapy vs. SEMS as 1st line treatment deployed by Endoscopic (ECP)

Tarantino I ${ }^{(15)} 2012$

P: Liver Trasplantation Complications

I: SEMS after endoscopic therapy failure

C: Self-expandable metallic stents

O: Success, Recurrence/ SEMS migration, Duration

\begin{tabular}{|c|c|c|c|}
\hline & SEMS after failure (\%) & SEMS 1st line(\%) & $P$ \\
\hline Success & $28 / 39(71.8)$ & $8 / 15(53.3)$ & NA* \\
\hline Failure & $11 / 39$ & $7 / 15$ & NA \\
\hline Recurrence & $4 / 28(14.3)$ & $2 / 8(25)$ & NA \\
\hline Migration & $13 / 39(33.3)$ & $7 / 15(46.7)$ & NA \\
\hline Follow up period (days) & $663 \pm 300$ & $432 \pm 66$ & NA \\
\hline
\end{tabular}

*NA - not available

1. Failure

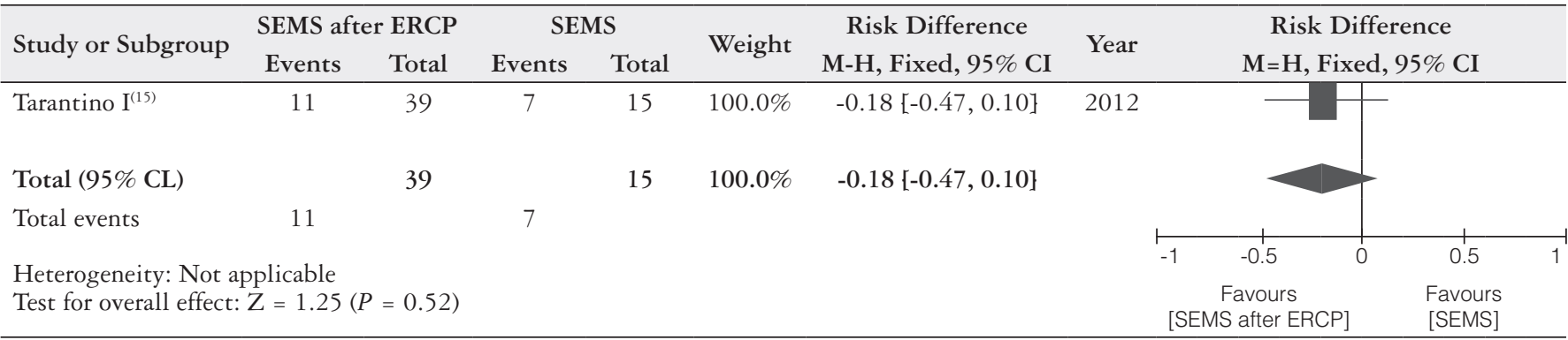

\section{Recurrence}

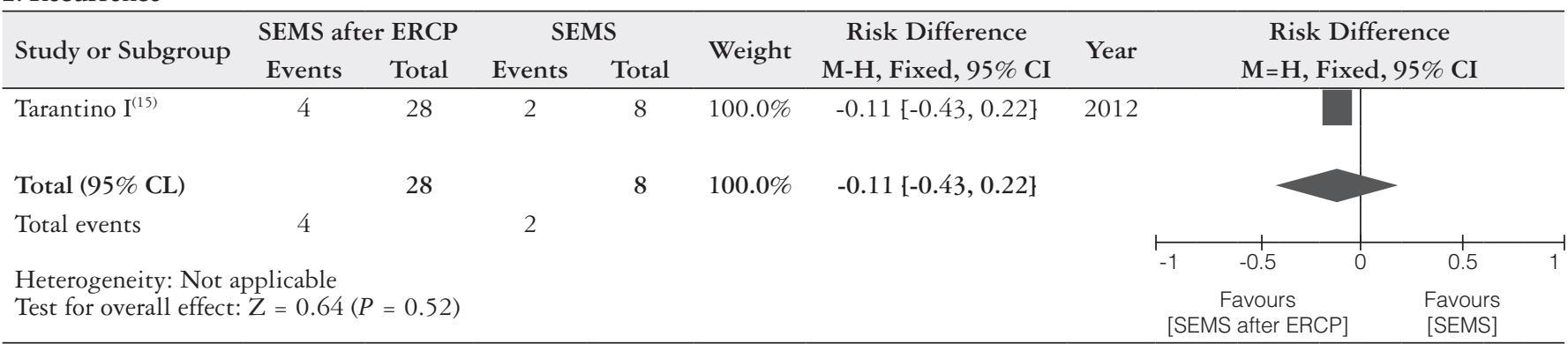

\section{Migration}

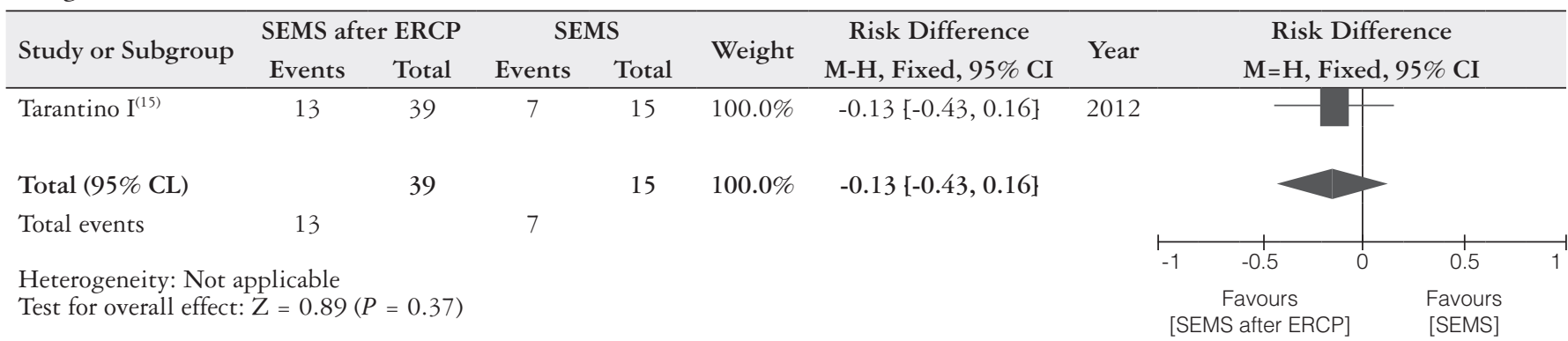

4. Follow up period

\begin{tabular}{|c|c|c|c|c|c|c|c|c|c|c|c|c|c|c|}
\hline \multirow{3}{*}{$\begin{array}{l}\begin{array}{l}\text { Study or } \\
\text { Subgroup }\end{array} \\
\text { Tarantino I }{ }^{(15)}\end{array}$} & \multicolumn{3}{|c|}{ SEMS after ERCP } & \multicolumn{3}{|c|}{ SEMS } & \multirow{2}{*}{ Weight } & \multirow{2}{*}{$\begin{array}{c}\text { Risk Difference } \\
\text { M-H, Fixed, 95\% CI }\end{array}$} & \multirow{2}{*}{ Year } & \multirow{2}{*}{\multicolumn{5}{|c|}{$\begin{array}{c}\text { Risk Difference } \\
\text { M=H, Fixed, 95\% CI }\end{array}$}} \\
\hline & \multirow{2}{*}{$\frac{\text { Mean }}{663}$} & \multirow{2}{*}{$\frac{S D}{300}$} & \multirow{2}{*}{$\frac{\text { Total }}{28}$} & \multirow{2}{*}{$\frac{\text { Mean }}{432}$} & \multirow{2}{*}{$\frac{\mathrm{SD}}{8}$} & \multirow{2}{*}{$\frac{\text { Total }}{15}$} & & & & & & & & \\
\hline & & & & & & & $100.0 \%$ & 231.00 [119.81, 342.19] & 2012 & & & & & \\
\hline Total (95\% CL) & & & 28 & & & 15 & $100.0 \%$ & 231.00 [119.81, 342.19] & & & & & & \\
\hline Heterogeneity & pplic & & & & & & & & & -1000 & -500 & 0 & 500 & 10 \\
\hline Test for overall & $: Z=4$ & (2) & $0.001)$ & & & & & & & [SEM & $\begin{array}{l}\text { Favou } \\
\text { S after }\end{array}$ & & $\begin{array}{l}\text { Favours } \\
\text { [SEMS] }\end{array}$ & \\
\hline
\end{tabular}


TABLE 4. Plastic stent vs SELF-expandable metallic stents (SEMS) deployed by percutaneous route (PTC)

\begin{tabular}{lccc}
\hline Kim J(6) 2010 & & & \\
P: Biliary anastomotic strictures following LDLT & & & \\
I: BD + Drainage catheter placement & & & \\
C: Covered retrievable stent placement after PTBD & & SEMS & \multicolumn{1}{c}{} \\
O: Success, Recurrence / Migration, Duration & Plastic stent & $14 / 20$ & 0.005 \\
\hline & $37 / 39$ & $6 / 20$ & NA* \\
\hline Success & $2 / 39$ & $12 / 20$ & 0.011 \\
Failure & $6 / 39$ & $1 / 14$ & n.s. \\
Complications & $4 / 37$ & $197 \pm 89$ & 0.018 \\
Recurrence & $278 \pm 115$ & & \\
Treatment duration (days) & & & \\
\hline
\end{tabular}

NA* - not available. LDLT: living donor liver transplantation; PTBD: percutaneous biliary drainage

(Table 1). Successful intervention rates $(60.0 \%$ in ECP vs $59.3 \%$ in PCP, $P=1.00)$ and time to recurrence after successful intervention $(44.8 \pm 7.4$ months in ECP vs $41.9 \pm 3.4$ months in PCP, $P=0.47$ ) were no different between these two techniques. However, the number of intervention sessions for PCP $(7.2 \pm 0.6)$ was higher than for ECP $(2.9 \pm 0.6)(P<0.01)$. Complication rate was similar between the groups $(24 \%$ in ECP vs $23 \%$ in PCP patients) ${ }^{(10)}$.

\section{Balloon dilation associated with Endoprothesis $(B D+E P)$ vs Ballon dilatation alone (BD)}

Two studies comparing BD+EP vs BD alone were included in the analysis ${ }^{(9,17)}$. The study by Zoepf et al. ${ }^{(18)}$ was excluded because the authors included only ischemic biliary lesions. It was possible to extract the data from patients with anastomotic strictures included in the studies by Zoepf et al. ${ }^{(17)}$ and Kulaksiz et al. ${ }^{(9)}$. Both studies described a high initial clinical success with $\mathrm{BD}+\mathrm{EP}$ and $\mathrm{BD}$ alone. However one study found a higher recurrence rate in the BD alone group. The meta-analysis showed no difference for the two techniques regarding initial clinical success, failure, complications or recurrence rates (Table 2).

\section{Self-expandable metallic stents (SEMS) as salvage therapy vs SEMS as 1st line treatment deployed by ECP}

One study compared the results of fully covered selfexpandable metallic stents used as salvage therapy after failure of the treatment with plastic stents versus the use of SEMS as the 1st line therapy of ABS post-OLT ${ }^{(15)}$. The clinical success, the recurrence, the failure and migration rates were no different between the two groups. However the follow up period was shorter in the SEMS as 1st line therapy group (Table 3).

\section{Plastic Stent vs SEMS deployed by PTC}

One study compared the results of plastic stents vs SEMS both deployed by PTC for the treatment of ABS post-living donor liver transplantation ${ }^{(6)}$. The clinical success and complication rates favored the plastic stent group. However, the treatment duration was shorter in the SEMS group (Table 4).

\section{DISCUSSION}

Biliary strictures after OLT are recognized as the Achilles' heel of OLT. They are usually classified in anastomotic biliary strictures (ABS) and non-anastomotic biliary strictures (NABS). The involved etiological factors seems to be the small bile duct diameter, a big disproportion between graft and recipient bile ducts, the ischemia time, tension on the anastomosis, infection and other technical issues ${ }^{(8)}$. On the other hand, ischemia related to hepatic artery problems seems to be more related to the occurrence of $\operatorname{NABS}^{(2,13)}$. NABS can present as a single or multiple stenosis and has a reserved prognosis when compared to $\mathrm{ABS}^{(18)}$. That is the reason why the present systematic review focused on ABS. We noticed that sometimes the two conditions were included in the cohort of patients but their results were not presented separately making impossible to evaluate the results of the treatment adequately.

Multiple sessions of balloon dilation followed by the endoscopic placement of plastic stents is the most commonly adopted strategy for the treatment of ABS after $\mathrm{OLT}^{(5)}$. The present systematic review makes clear that there is a paucity of well-designed randomized controlled trials to support this strategy. It is possible that several centers have used their expertise and experience in treatment of iatrogenic lesions of the biliary tree, and they may have brought the same concept for the treatment of ABS after OLT.

Concerning the route of access, there is only one retrospective comparative trial comparing the transhepatic and the endoscopic accesses ${ }^{(10)}$. In both groups the treatment was based on several sessions of dilation and stent insertion. There were no significant differences except for the fact that more sessions were needed in the transhepatic group. The authors attribute this occurrence to the accidental dislodgement of the external tube when the patients were at home. In this study, non-anastomotic biliary stricture cases occurred roughly in $30 \%$ of both groups. The authors recognized nonanastomotic biliary stricture as a predictor of failure of the dilation/stenting treatment. Considering that the transhepatic route is more invasive, and usually implicates in external drainage at some point of the treatment, it is easy to understand the widespread preference for the endoscopic access. 
Two studies compared balloon dilatation versus balloon dilatation with plastic stenting for the treatment of ABS after $\operatorname{OLT}^{(9,17)}$. One of them ${ }^{(9)}$ was initiated as a prospective randomized trial (registration at ClinicalTrials.gov NCT00487201). As some of the patients were not randomized the authors changed the trial design to a prospective comparative study. The initial clinical success and sustained clinical success rates were similar, around $95 \%$ and $70 \%$, respectively. More complication events were observed in the stent group. The main reason was the occurrence of stent migration and occlusion. The other study ${ }^{(17)}$ was a retrospective comparative one. Initial success rates were similar in both groups, around $90 \%$. However in the balloon dilatation group stenosis recurrence rate was higher when compared to the combo treatment arm, roughly $60 \%$ and $30 \%$ respectively. The complication rates were similar between the groups. The present meta-analysis did not show any difference between the initial or sustained clinical success rates or the complication event rate when balloon dilatation and balloon dilatation plus stenting were compared. Some technical aspects may explain the above-mentioned results. In the study by Kulaksiz et al. ${ }^{(9)}$, the mean number of sessions of endoscopic treatment was similar in both groups (five sessions) while in the study by Zoepf et al. ${ }^{(17)}$, the mean number of sessions was double in the combo treatment group (four sessions) when compared to the balloon group (two sessions). Additionally, Kulaksiz et al. ${ }^{(9)}$ dilated the ABS up to $6 \mathrm{~mm}(18 \mathrm{Fr})$ and placed just a $10 \mathrm{Fr}$ stent in the first session which could explain the higher complication rate which was due to stent migration in the combo treatment group. Finally, Zoepf et al. ${ }^{(17)}$ did not included stent migration as a complication. Taking in consideration these results it is possible that an intensive endoscopic treatment with several sessions of balloon dilation may achieve similar results when compared to balloon dilation and plastic stenting. If this were proved in a well-designed randomized trial, balloon dilation would be probably more cost effective in the treatment of ABS after OLT. On the other hand one could underline the fragility of the results obtained by the metanalytic model in experimental trials involving invasive procedures because the technical nuances (i.e., expertise of the operator, number of stents, dilatation pressure, duration of dilatation) are hardly controlled in that statistical model. In addition, another limitation of the results obtained by the present metanalysis was due to the scarce number of included studies and the small number of patients.

The need for several sessions of endoscopic treatment for the treatment of ABS after OLT make the rationale for the use of metallic stents which achieve a higher diameter and develop later biliary obstruction when compared to plastic stents. The first series employed partially covered stents which presented lower migration rates at the cost of embedding of the uncovered mesh into the duct wall making its removal difficult and causing irreversible inflammatory changes in the bile duct ${ }^{(5)}$. More recently fully covered metallic stents were employed with higher migration rates and variable results ${ }^{(5)}$. We found a systematic review comparing the use of multiple plastic stents with self-expandable metallic stents for the treatment of ABS after OLT ${ }^{(5)}$. The authors recognize that there were no well-designed randomized trials, but only small case series for both treatment strategies, making impossible a meta-analysis. They also conclude that the success rates with multiple plastic stents are possibly higher when the patients are treated for 12 months or longer. They found the same trend in patients treated with metallic stents for 3 months or longer.

We found two retrospective comparative studies comparing plastic and metallic stents ${ }^{(6,15)}$. Tarantino et al. ${ }^{(15)} \mathrm{com}$ pared the outcomes of patients with failure of the multiple plastic stenting strategy sent to metallic stenting (salvage therapy group) with patients sent directly to metallic stenting as a first line treatment (first line treatment group). The authors showed that the sustained clinical success rate was higher in the salvage therapy group when compared to the first line therapy group. The authors left the metallic stents for only 2 months in both groups. Kim et al. ${ }^{(6)}$ compared plastic and metallic stents both placed by percutaneously in patients with biliary strictures submitted to living donor liver transplantation. The sustained clinical success was higher in the plastic group ( 95 vs $70 \%, P<0.0005$ ). It must be considered that the metallic stent was left in place for just 2 months.

These findings raise the concern about the lack of adequate evidence to support the early adoption of new technology in clinical practice based just on theoretical concepts and on previous experience with similar situation (i.e. endoscopic treatment of iatrogenic biliary strictures). As mentioned above, it is not proved that the combo treatment (balloon + plastic stent) is superior to balloon dilatation alone. It is possible that there are patients who would have resolution of the ABL with balloon dilatation alone. Well-designed randomized controlled trials could help to identify them.

It is also noteworthy that a search for ongoing randomized controlled trials at the site http://clinicaltrials.gov revealed that there are four trials comparing fully covered metallic stents with plastic stents for the treatment of ABS after OLT, three of them still recruiting patients. Most of them are supposed to collect the first data by the end of this year or the beginning of 2014.

In conclusion, there is a vacuum in the literature of the endoscopic management of ABL after OLT. Well-designed randomized trials comparing balloon dilation vs multiple plastic stenting vs fully covered metallic stents are warranted to define a more tailored approach to this population.

\section{Author contribution}

Nacif LS and Bernardo WM design and performed the research. Bernardo L and Bernardo WM performed the statistical analysis and meta-analysis; Nacif LS, Andraus W, Torres L and Maluf-Filho F drafted the article and D'Albuquerque LC, Chaib E and Maluf-Filho F revising it critically and all authors approved the final version to be published. 
Nacif LS, Bernardo WM, Bernardo L, Andraus W, Torres L, Chaib E, D’Albuquerque LC, Maluf-Filho F. Tratamento endoscópico na estenose da anastomose biliar no pós-transplante hepático: revisão sistemática e meta-análise. Arq Gastroenterol. 2014,51(3):240-9.

RESUMO - Contexto - A estenose biliar figura como uma das complicações mais frequentes pós-transplante hepático. São classificadas em anastomóticas e não anastomóticas, sendo estas últimas geralmente isquêmicas. Dentre as várias opções de tratamento, destacam-se a dilatação balonada, a colocação de próteses plásticas e a colocação de próteses metálicas autoexpansíveis, que podem ser realizadas tanto por via endoscópica, como por via percutânea. Não há consenso quanto ao melhor tratamento para a estenose da anastomose biliar. Objetivo - O objetivo deste estudo foi realizar revisão sistemática da literatura sobre o resultado do tratamento endoscópico da estenose da anastomose biliar após transplante hepático. Método - Revisão sistemática da literatura foi realizada sobre o tratamento da estenose da anastomose biliar pós transplante hepático, através do acesso aos bancos de dados pesquisados eletronicamente: Medline - PubMed, EMBASE, Scielo- Lilacs, Cochrane de janeiro de 1966 a abril de 2013. Resultados - Não foi encontrado estudo clinico controlado e randomizado. A maioria dos estudos são comparativos retrospectivos ou prospectivos. Um estudo (86 pacientes) avaliou o acesso endoscópico e percutâneo. As taxas de sucesso clínico sustentados foram semelhantes, mas a duração do tratamento foi maior no grupo de acesso percutâneo. Dois estudos (56 pacientes) compararam a dilatação por balão com dilatação por balão e múltiplas próteses plásticas. Não houve diferenças em relação as taxas de sucesso e de complicações clínicas sustentadas. Conclusão - A dilatação com balão é tão eficaz quanto a dilatação associada à colocação de múltiplas próteses plásticas para a resolução da estenose da anastomose biliar. Estudos randomizados bem desenhados ainda são necessários para comparar a simples dilatação balonada e a colocação de próteses plásticas.

DESCRITORES - Transplante de fígado. Constrição. Revisão. Metanálise. Endoscopia gastrointestinal.

\section{REFERENCES}

1. Chang JH, Lee IS, Chun HJ, Choi JY, Yoon SK, Kim DG, et al. Comparative study of rendezvous techniques in post-liver transplant biliary stricture. World J Gastroenterol. 2012;18:5957-64.

2. Colonna JO, Shaked A, Gomes AS, Colquhoun SD, Jurim O, McDiarmid SV, et al. Biliary strictures complicating liver transplantation. Incidence, pathogenesis, management, and outcome. Ann Surg. 1992;216:344-50; discussion 50-2.

3. Giampalma E, Renzulli M, Mosconi C, Ercolani G, Pinna AD, Golfieri R. Outcome of post-liver transplant ischemic and nonischemic biliary stenoses treated with percutaneous interventions: the Bologna experience. Liver Transpl. 2012;18:177-87.

4. Gómez CM, Dumonceau JM, Marcolongo M, de Santibañes E, Ciardullo M, Pekolj J, et al. Endoscopic management of biliary complications after adult living-donor versus deceased-donor liver transplantation. Transplantation. 2009;88:1280-5.

5. Kao D, Zepeda-Gomez S, Tandon P, Bain VG. Managing the post-liver transplantation anastomotic biliary stricture: multiple plastic versus metal stents: a systematic review. Gastrointest Endosc. 2013;77:679-91.

6. Kim J, Ko GY, Sung KB, Gwon DI, Lee SG, Kim KM, et al. Percutaneously placed covered retrievable stents for the treatment of biliary anastomotic strictures following living donor liver transplantation. Liver Transpl. 2010;16:1410-20.

7. Kobayashi N, Kubota K, Shimamura T, Watanabe S, Kato S, Suzuki K, et al. Complications of the treatment of endoscopic biliary strictures developing after liver transplantation. J Hepatobiliary Pancreat Sci. 2011;18:202-10.

8. Koneru B, Sterling MJ, Bahramipour PF. Bile duct strictures after liver transplantation: a changing landscape of the Achilles' heel. Liver Transpl. 2006; $12: 702-4$.

9. Kulaksiz H, Weiss KH, Gotthardt D, Adler G, Stremmel W, Schaible A, et al. Is stenting necessary after balloon dilation of post-transplantation biliary strictures? Results of a prospective comparative study. Endoscopy. 2008;40:746-51.
10. Lee SH, Ryu JK, Woo SM, Park JK, Yoo JW, Kim YT, et al. Optimal interventional treatment and long-term outcomes for biliary stricture after liver transplantation. Clin Transplant. 2008;22:484-93.

11. Li QY, Qin YS, Ling Q, Yang FC, Zheng SS. No therapeutic ERCP in anastomotic stricture without intrahepatic biliary dilation after liver transplantation. Hepatogastroenterology. 2011;58:1127-31.

12. Pasha SF, Harrison ME, Das A, Nguyen CC, Vargas HE, Balan V, et al. Endoscopic treatment of anastomotic biliary strictures after deceased donor liver transplantation: outcomes after maximal stent therapy. Gastrointest Endosc. 2007;66:44-51.

13. Sommacale D, Aoyagi T, Dondero F, Sibert A, Bruno O, Fteriche S, et al. Repeat endovascular treatment of recurring hepatic artery stenoses in orthotopic liver transplantation. Transpl Int. 2013;26:608-15.

14. Tabibian JH, Asham EH, Han S, Saab S, Tong MJ, Goldstein L, et al. Endoscopic treatment of postorthotopic liver transplantation anastomotic biliary strictures with maximal stent therapy (with video). Gastrointest Endosc. 2010;71:505-12.

15. Tarantino I, Mangiavillano B, Di Mitri R, Barresi L, Mocciaro F, Granata A, et al. Fully covered self-expandable metallic stents in benign biliary strictures: a multicenter study on efficacy and safety. Endoscopy. 2012;44:923-7.

16. Wells GA, Shea B, O'Connell D, Peterson J, Welch V, Losos M, Tugwell P. The Newcastle-Ottawa Scale (NOS) for Assessing the Quality of Nonrandomised Studies in Meta-analyses. [Internet] Ottawa: Ottawa Hospital Research Institute. [cited 2013 April]. Available from: http://www.ohri.ca/programs/clinical_epidemiology/oxford.asp

17. Zoepf T, Maldonado-Lopez EJ, Hilgard P, Malago M, Broelsch CE, Treichel $\mathrm{U}$, et al. Balloon dilatation vs. balloon dilatation plus bile duct endoprostheses for treatment of anastomotic biliary strictures after liver transplantation. Liver Transpl. 2006;12:88-94.

18. Zoepf T, Maldonado de Dechêne EJ, Dechêne A, Malágo M, Beckebaum S, Paul A, et al. Optimized endoscopic treatment of ischemic-type biliary lesions after liver transplantation. Gastrointest Endosc. 2012;76:556-63.

Received 3/3/2014 Accepted 14/4/2014 